Research Article

\title{
Objective Evaluation of Drivability in Passenger Cars with Dual-Clutch Transmission: A Case Study of Static Gearshift Condition
}

\author{
Wei Zhou $\mathbb{D}^{1,2}$ Xuexun Guo, ${ }^{1,2}$ Xiaofei Pei $\mathbb{D}^{1,2}$ Chengcai Zhang $\mathbb{D}^{1,2}$ Jun Yan, ${ }^{3}$ \\ and Jialei $\mathrm{Xia}^{3}$ \\ ${ }^{1}$ Hubei Key Laboratory of Advanced Technology of Automotive Parts, Wuhan University of Technology, Wuhan 430070, China \\ ${ }^{2}$ Hubei Collaborative Innovation Centre for Automotive Components Technology, Wuhan University of Technology, \\ Wuhan 430070, China \\ ${ }^{3}$ Powertrain Development Department, Dongfeng Motor Corporations Technical Centre, Wuhan 430058, China
}

Correspondence should be addressed to Chengcai Zhang; zhangchc@whut.edu.cn

Received 5 July 2020; Revised 18 September 2020; Accepted 8 October 2020; Published 30 October 2020

Academic Editor: Jun Shen

Copyright (c) 2020 Wei Zhou et al. This is an open access article distributed under the Creative Commons Attribution License, which permits unrestricted use, distribution, and reproduction in any medium, provided the original work is properly cited.

This paper is aimed at the problem that the subjective drivability evaluation by experienced test drivers is limited in time efficiency and is of high cost and poor repeatability. In this article, an intelligent drivability objective evaluation tool (I-DOET) for passenger cars with dual-clutch transmission (DCT) is developed and verified by real vehicle testing. First, the signal denoising method and its key parameters, which are suitable for drivability evaluation, are selected based on analytic hierarchy process (AHP) and technique for order preference by similarity to ideal solution (TOPSIS). Besides, combined with the uncertainty characteristics of subjective judgment, a mathematical model of the objective drivability evaluation FARODE (fuzzy AHP-RS based on objective drivability evaluation) is proposed by using the fuzzy comprehensive assessment (FCA) method. The AHP and rough set (RS) method are used to calculate the subjective and objective weights of the drivability evaluation, respectively, and the proportion of subjective and objective weights is determined by the principle of minimum relative information entropy. The fuzzy matrix is built by membership function of the evaluation indexes. Finally, the static gearshift condition focused on by the subjective evaluation experts is taken as a case study. The predictability score is obtained by combining the drivability quantization lever vector, comprehensive weight, and fuzzy matrix. The experimental results indicate that the proposed method is applicable for objective drivability evaluation in passenger cars with DCT.

\section{Introduction}

Drivability is essentially needed as one of important performances for vehicle manufacturers, and it is often evaluated based on subjective feelings of experienced test drivers [1]. However, these evaluations are time-consuming, costly, and nonrepeatable, and the reliability of the results is closely related to the psychological state of the evaluator and the test environment [2]. As an important supplement to subjective evaluation, the objective test method can not only express subjective feelings through objective indicators but also obtain more system information that is not subjectively perceptible, and it is easier to optimize vehicle performance [3]. Hence, the development of a reliable and efficient objective evaluation system plays an important role in improving the drivability of vehicles.

Several researchers have studied the objective evaluation of drivability for vehicles. As early as 2000 , drivability was defined as positive and negative acceleration response, comfortable pedal response, and accurate gear shifting to meet the normal performance expectations of drivers [4]. In 2007, a series of drivability indicators for engine start conditions were proposed by AVL, but only a few evaluation indicators were published [5]. Jayaraman et al. [6] constructed a drivability index system for 9 working conditions such as engine start, shut-off, starting, and full-throttle acceleration; however, only some evaluation indexes are given. Khodabakhshian et al. [7] proposed that the 
drivability of the vehicle can be improved by optimizing the shift strategy and established the mapping relationship between the evaluation index and the control parameters.

The objective evaluation system is mainly composed of a signal acquisition module and a data analysis module. The signal acquisition module is used to obtain vehicle status signals synchronously, including vehicle acceleration, vehicle speed, engine speed, engine torque, transmission gear, brake pedal signal, accelerator pedal signal and air conditioner switch signal, etc., depending on the determination of working conditions and the selection of objective indicators [8-10]. In the data analysis module, the method of multisource data fusion and sliding window is employed to convert the original data into a single working condition fragment, which can greatly overcome the shortcoming of relying on vague professional experience and inefficient manual operation [11]. In [8], the objective evaluation can be divided into 12 operation conditions, such as idle, engine start, tip-in, tip-out and static gearshift, etc.

In recent years, with the development and popularization of passenger cars with DCT, better shift quality has been demanded by drivers [12]. The purpose of drivability evaluation in static gearshift condition is to evaluate the comfort during the manual switching of $\mathrm{P} / \mathrm{R} / \mathrm{N} / \mathrm{D}$ by the driver [13]. Because the power transmission system is a multirotational inertia system, it is difficult to complete the static gearshift process instantaneously. The disturbance of the engine and the unreasonable control logic of the gearbox may cause different degrees of shock on the vehicle. When the vibration is severe, drivers and passengers will be left with an unbearable driving experience, which decreases the drivability evaluation. Therefore, it is necessary to find the main factors that affect the shift quality through the objective drivability evaluation and provide certain suggestions to calibration engineers.

In the objective evaluation of drivability, vehicle longitudinal acceleration is the main parameter of response characteristics and comfort evaluation, which has a great influence on the extraction of feature indexes $[10,14]$. Several researchers have studied the method of acceleration signal denoising $[8,15-22]$. However, the evaluation indexes and analysis methods are rarely studied.

Although Liu et al. [20, 21] proposed the selection of threshold rules for wavelet filtering by quantitative indicators such as root-mean-square error (RMSE), signal-tonoise ratio (SNR), and smoothness of signal (SS), the effect of denoising composite weights was not considered. Zhou et al. [8] also developed an evaluation model of denoising, combined with SNR, mean error (ME), RMSE, and SS, but they only focused on evaluating the denoising effect based on a single factor; it is difficult to choose a suitable denoising method and its key parameters. Hence, a comprehensive evaluation method is indispensable to be applied for the selection of denoising method and its key parameters.

Generally, reasonable indicators and evaluation methods are the prerequisites for constructing an objective evaluation system for drivability. The jerk, shock, engine speed fluctuation, stabilization duration, shift delay, and engine speed undershoot are proposed by Jauch et al., Koprubasi et al.,
Lakshmanan et al., and Winter et al. [12, 23-25]. The research on drivability evaluation has been discussed using various methods [26-30]. The TOPSIS method, neural networks (NN), FAHP, multihierarchical grey relational analysis, and support vector regression are applied for the development of the objective evaluation for drivability. However, the rules for formulating the weights are not considered. This is insufficient for the objective drivability evaluation model to guarantee the accurate for predict result.

The rest of the paper is organized as follows. The structure of objective evaluation for drivability and the test platform of I-DOET are designed in Section 2. The principle for determining the longitudinal acceleration signal denoising method and its key parameters are introduced in Section 3. The mathematical model of the objective drivability evaluation FARODE is developed in Section 4. In Section 5, combined with the static gearshift condition in real vehicle testing, the prediction accuracy of the proposed drivability evaluation model is analysed. Finally, the conclusions are summarized inSection 6.

\section{Structure of Objective Evaluation for Drivability}

Objective evaluation for drivability is a precise and effective evaluation of driving performance through scientific quantitative methods, which involves three main tasks, namely, parameter acquisition, index extraction, and performance prediction, as illustrated in Figure 1. The parameter acquisition layer collects vehicle state information through an acceleration sensor and an on-board CAN network. Using signal smoothing and denoising to process the original signal, the effective information could be obtained, which reflects the actual state of the vehicle. The index extraction layer reflects the driver's subjective feelings (e.g., comfort and response) through quantitative indicators. And then, based on operating condition characteristics and the multisource signal (e.g., engine speed, pedal, and gear) provided by the parameter acquisition layer, the index extraction layer identifies the current conditions (e.g., gear shift, engine start, idle, constant speed, and tip-in) and calculates the corresponding objective characteristic index. Finally, in order to evaluate the objective drivability of the vehicle, the performance prediction layer uses an evaluation model to calculate the index weights, and at the same time, the drivability score and its optimization items can be obtained objectively and steadily.

In order to achieve the above tasks, a set of I-DOET is developed, which includes 4 ADC acquisition modules, a vehicle CAN signal acquisition module, and a software analysis module. In [23], vehicle longitudinal dynamics theory and calibration engineer experience are combined to show that longitudinal acceleration, vehicle speed, engine speed, engine torque, transmission gear, accelerator pedal opening, and braking signal are the main parameters of drivability. The longitudinal acceleration signal is obtained through ADC module in the I-DOET platform, and the vehicle speed, engine speed, engine torque, transmission gear position, accelerator pedal opening, and braking signal 


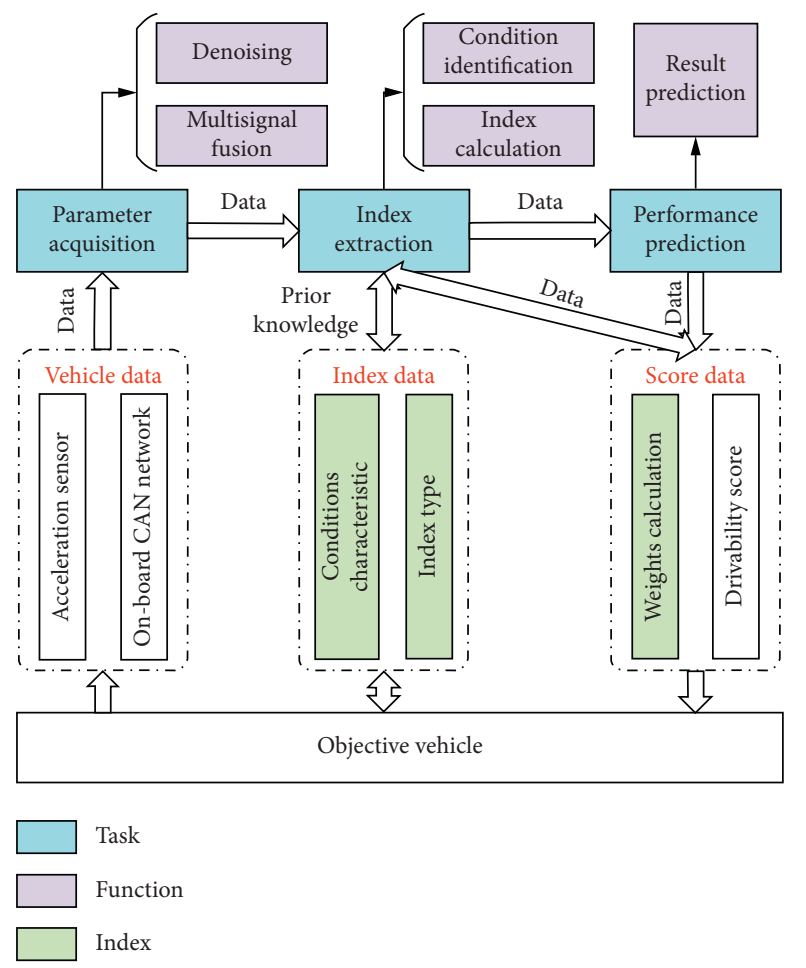

FIgURE 1: The structure of objective evaluation for drivability.

can be obtained through CAN module. The functional modules of the software platform are described in Sections $3-5$.

\section{The Denoising Quality Evaluation Based on the AHP-TOPSIS Model}

The longitudinal acceleration signal of the vehicle is one of the main parameters that determine the accuracy of the feature point extraction of the objective evaluation. Due to the random vibration caused by uneven road surface and vehicle suspension, and the fact that the acceleration sensor is easily interfered by the electromagnetic field, the acceleration signal shows nonlinearity and instability, which contains a certain amount of noise, making it difficult to take both denoising and smoothing into account. Therefore, multiple indicators need to be selected to analyse the quality of denoising.

3.1. Evaluation Indexes of Denoising Quality. The denoising method of longitudinal acceleration signal suitable for the drivability objective evaluation not only needs to retain the true value of the signal but also needs to have smooth features to ensure the accuracy and reliability of the feature point recognition. The ME, RMSE, SNR, and SS are commonly used evaluation indicators [8]. Among them, the ME is the average value of the difference between the filtered signal and the ideal acceleration signal, which is obtained by

$$
\mathrm{ME}=\frac{1}{n} \sum_{i=1}^{n}|r(i)-s(i)|
$$

where $n$ is the number of signal points and $r(i)$ and $s(i)$ are the ideal acceleration signal and the filtered acceleration signal, respectively.

The RMSE can be used to express small deviations and significant deviations, and the precision of the filtering effect can be given by

$$
\mathrm{RMSE}=\sqrt{\frac{1}{n} \sum_{i=1}^{n}(r(i)-s(i))^{2}}
$$

The SNR is the ratio of the average power for signals and the average power for noises. The larger the value, the more effective the components of the signal characteristic. It is represented by

$$
\operatorname{SNR}=10 \lg \left(\frac{\sum_{i=1}^{n} f^{2}(i)}{\sum_{i=1}^{n}[f(i)-s(i)]^{2}}\right)
$$

where $f(i)$ is the acceleration signal with noise.

The sudden changes and spikes of the signal will greatly interfere with the accuracy of the evaluation index extraction. The SS is obtained by the ratio of the sum for the signal after denoising and the variance of the original signal. It is calculated by

$$
\mathrm{SS}=\frac{\sum_{i=2}^{n}[s(i)-s(i-1)]^{2}}{\sum_{i=2}^{n}[f(i)-f(i-1)]^{2}} .
$$

In order to evaluate the quality of denoising more accurately, the signal-to-noise ratio gain (SNRG) and correlation coefficients (CC) have been proposed [31, 32]. The SNRG is an important indicator to measure random vibration. It can be given by

$$
\text { GSNR }=\log \left(\frac{\sum_{i=1}^{n} f^{2}(i)}{\sum_{i=1}^{n}[f(i)-s(i)]^{2}}\right)\left[\frac{\sum_{i=1}^{n} f^{2}(i)}{\sum_{i=1}^{n}[f(i)-r(i)]^{2}}\right] .
$$

The CC is used to characterize the approximate relationship between the filtered acceleration signal and the ideal acceleration signal, which is obtained by

$$
\mathrm{CC}=\frac{\sum_{i=1}^{n}(s(i)-\bar{s})(r(i)-\bar{r})}{\sqrt{\sum_{i=1}^{n}(s(i)-\bar{s})^{2} \sum_{i=1}^{n}(r(i)-\bar{r})^{2}}}
$$

where $\bar{s}$ and $\bar{r}$ are the mean value of filtered acceleration signal and the mean value of the ideal acceleration signal, respectively.

According to the requirement of denoising, the filtered signal should be closer to the ideal signal and smoother. The quantitative index system for denoising of the longitudinal acceleration signal is shown in Table 1 .

3.2. AHP-TOPSIS Evaluation Model. AHP is a method of dealing with complex and constrained multifactor systems through expert experience. It can be used to plan the hierarchical structure clearly and assign the weight of factors scientifically. TOPSIS is a method for solving multiattribute 
Table 1: The quantitative index system for denoising.

\begin{tabular}{lcc}
\hline Macro index layer & Micro index layer & Index type \\
\hline \multirow{3}{*}{ Signal error } & ME & I \\
& RMSE & I \\
& SNR & II \\
Smoothing & SNG & II \\
\hline
\end{tabular}

Index type division rules: $\mathrm{I}$ is the smaller the better type; II is the larger the better type.

decision-making schemes. It often uses ideal and anti-ideal solutions to measure the order of evaluation schemes. Integrating the evaluation model of AHP-TOPSIS can find the goal that meets the intention of the decision maker from the decision object group scientifically and effectively. The flowchart is shown in Figure 2.

First, based on the experience of experts, a binary comparison method is used to compare and assign different indicators at the same level. The assignment criteria are listed in Table 2, and the judgment matrix $X$ is constructed:

$$
X=\left[\begin{array}{cccccc}
X_{11} & X_{12} & \cdots & X_{1 j} & \cdots & X_{1 n} \\
X_{21} & X_{22} & \cdots & X_{2 j} & \cdots & X_{2 n} \\
\vdots & \vdots & \ddots & \vdots & \ddots & \vdots \\
X_{i 1} & X_{i 2} & \cdots & X_{i j} & \cdots & X_{i n} \\
\vdots & \vdots & \ddots & \vdots & \ddots & \vdots \\
X_{n 1} & X_{n 2} & \cdots & X_{n j} & \cdots & X_{n n}
\end{array}\right],
$$

The square root method is used to obtain the normalization and maximum feature root of the judgment matrix. The relative weight of each factor is obtained from the maximum feature root and its feature vector:

$$
\left\{\begin{array}{l}
A_{i}=\prod_{j=1}^{n} X_{i j}, \\
B_{i}=\frac{\sqrt[n]{A_{i}}}{\sum_{i=1}^{n} \sqrt[n]{A_{i}}}, \\
\lambda_{\max }=\sum_{i=1}^{n} \frac{(X B)_{i}}{n B_{i}}, X w=\lambda_{\max } \times w,
\end{array}\right.
$$

where $A$ and $B$ are the result of multiplication of the comparison matrix by row and the normalized vector, respectively, and $\lambda_{\max }$ and $w$ are the largest feature root and the weight of each factor, respectively.

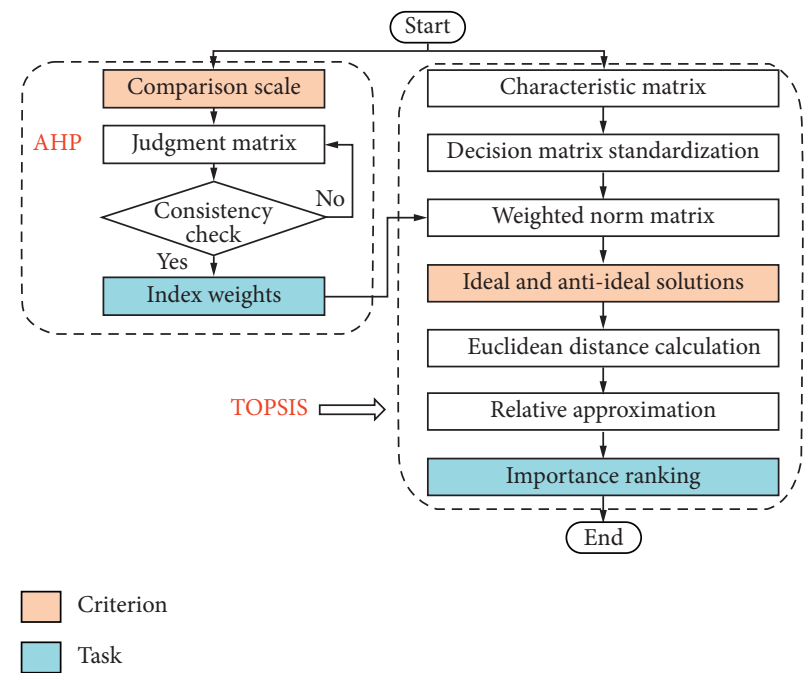

FIGURE 2: Flowchart of AHP-TOPSIS evaluation.

The consistency check of AHP is used to analyse the rationality of judgment matrix, which directly affects the reliability of the AHP output. The random index (RI) can be searched in Table 3, and the consistency index (CI) and consistency ratio (CR) can be obtained by

$$
\left\{\begin{array}{l}
\mathrm{CI}=\frac{\lambda_{\max }-n}{n}, \\
\mathrm{CR}=\frac{\mathrm{CI}}{\mathrm{RI}} .
\end{array}\right.
$$

The upper limit for CR is 0.1. If CR exceeds 0.1, the judgment matrix should be reestablished and the index weights should be calculated until the consistency check is satisfied.

TOPSIS method can incorporate important weights of criteria into the comparison procedures to provide an understandable and rational ranking result, which consists of the following seven steps.

Step 1. An initial matrix $D$ for evaluation is established by

$$
D=\left[\begin{array}{ccccc}
D_{11} & \cdots & D_{1 l} & \cdots & D_{1 q} \\
\vdots & \ddots & \vdots & \ddots & \vdots \\
D_{k 1} & \cdots & D_{k l} & \cdots & D_{k q} \\
\vdots & \ddots & \vdots & \ddots & \vdots \\
D_{p 1} & \cdots & D_{p l} & \cdots & D_{p q}
\end{array}\right],
$$

where $p$ and $q$ are the number of schemes and indexes, respectively, and $D_{k l}$ is a crisp value indicating the performance rating of each alternative $D_{k}$ with respect to each criterion $D_{l}, k=1,2, \ldots, p ; l=1$, $2, \ldots, q$.

Step 2. The standardized decision matrix $S$ is adopted by 
TABLE 2: Comparable scale of 1 9.

\begin{tabular}{lcc}
\hline Scale & Definition & Notation \\
\hline 1 & Equally important & $X_{i}$ is equally important to $X_{j}$ \\
3 & Slightly important & $X_{i}$ is moderately more important than $X_{j}$ \\
5 & Strongly important & $X_{i}$ is strongly more important than $X_{j}$ \\
7 & Very important & $X_{i}$ is very strongly more important than $X_{j}$ \\
9 & Extremely important & $X_{i}$ is extremely more important than $X_{j}$ \\
$2,4,6,8$ & Intermediate values of the above judgment & \\
\hline
\end{tabular}

TABLE 3: Values of average stochastic coincidence indicators.

\begin{tabular}{cccccccccccc}
\hline$n$ & 1 & 2 & 3 & 4 & 5 & 6 & 7 & 8 & 9 & 10 & 11 \\
\hline RI & 0 & 0 & 0.58 & 0.90 & 1.12 & 1.24 & 1.32 & 1.41 & 1.45 & 1.49 & 1.51 \\
\hline
\end{tabular}

$$
S=\left(S_{k l}\right)_{p \times q}=\left[\begin{array}{ccccc}
S_{11} & \cdots & S_{1 l} & \cdots & S_{1 q} \\
\vdots & \ddots & \vdots & \ddots & \vdots \\
S_{k 1} & \cdots & S_{k l} & \cdots & S_{k q} \\
\vdots & \ddots & \vdots & \ddots & \vdots \\
S_{p 1} & \cdots & S_{p l} & \cdots & S_{p q}
\end{array}\right],
$$

where the $S_{k l}$ value is calculated by

$$
S_{k l}=\frac{D_{k l}}{\sqrt{\sum_{k=1}^{p} D_{k l}^{2}}}
$$

Step 3. The weighted standardized decision matrix $R$ is given by

$$
R=\left(R_{k l}\right)_{p \times q}=\left[\begin{array}{ccccc}
R_{11} & \cdots & R_{1 l} & \cdots & R_{1 q} \\
\vdots & \ddots & \vdots & \ddots & \vdots \\
R_{k 1} & \cdots & R_{k l} & \cdots & R_{k q} \\
\vdots & \ddots & \vdots & \ddots & \vdots \\
R_{p 1} & \cdots & R_{p l} & \cdots & R_{p q}
\end{array}\right],
$$

where the $R_{k l}$ value is calculated by

$$
R_{k l}=S_{k l} * w_{l}
$$

where $w_{l}$ represents the weight of the lth criterion.

Step 4. The index attributes applicable to TOPSIS are divided into benefit criteria $(B)$ and cost criteria $(C)$, and the ideal solution $R_{l}^{+}$and anti-ideal solution $R_{l}^{-}$are obtained by

$$
\left\{\begin{array}{l}
R_{l}^{+}=\left\{\left(\max R_{k l} \mid l \in B\right),\left(\min R_{k l} \mid l \in C\right)\right\} \\
R_{l}^{-}=\left\{\left(\max R_{k l} \mid l \in C\right),\left(\min R_{k l} \mid l \in B\right)\right\}
\end{array}\right.
$$

Step 5. The separation measures of each alternative from the ideal solution and anti-ideal solution are calculated by
$\left\{E_{k}^{+}=\sqrt{\sum_{l=1}^{q}\left(R_{k l}-R_{l}^{+}\right)^{2}}, E_{k}^{-}=\sqrt{\sum_{l=1}^{q}\left(R_{k l}-R_{l}^{-}\right)^{2}}\right.$.

where $E_{k}^{+}$and $E_{k}^{-}$are the Euclid distance of each alternative from the ideal solution and anti-ideal solution, respectively.

Step 6. The relative approximation to the ideal solution $E_{k}$ is obtained by

$$
E_{k}=\frac{E_{k}^{-}}{E_{k}^{-}+E_{k}^{+}}
$$

Step 7. The important ranking of the performance order is given by $E_{k}$ index value, which lies between 0 and 1 . The larger the index value, the better the performance of the alternatives.

\subsection{Application of Longitudinal Acceleration Signal Denoising} Based on AHP-TOPSIS. According to the previous work [4], firstly, the acceleration signal with noise is taken as the research objective, and then the ME, RMSE, SNR, SNRG, SS, and $\mathrm{CC}$ are selected as index layers to take the best scheme of denoising. AHP-TOPSIS is used to select the appropriate denoising method and its key parameters. In order to calculate the weight of each characteristic index more reliably, three experts with rich experience in signal denoising are invited in the questionnaire survey to discuss the importance of different evaluation indexes on the signal denoising effect. The 1-9 scale method to compare the importance of evaluation indicators for experts is selected, which is described in Table 2. Table 4 is the judgment matrix of the target layer.

According to equations (7)-(10), $\lambda_{\max }=6.125$, $\mathrm{CI}=0.0249, \mathrm{RI}=1.24, \mathrm{CR}=0.020<0.1$, which satisfies the test requirements for judging the consistency of the matrix. The weight of each index is $w=[0.036,0.044,0.428,0.275$, $0.150,0.067]$. Then, TOPSIS is used to rank the schemes, and the proposed AHP-TOPSIS decision model is used to select the appropriate denoising method and its key parameters. After obtaining the local index weights through AHP, the decision matrix is established.

In the research, $5 \mathrm{~Hz}$ low-pass filtering, 21-point smoothing filter, and Db6, Coif5, Sym3, Sym4, and Sym6 wavelet threshold as the basis function are selected as the research objective and calculated by equations (1)-(6), the calculation results of ME, RMSE, SNR, SNRG, SS, and CC are shown in Table 5. And these values are used as the initial 
TABLE 4: The judgment matrix of the denoising.

\begin{tabular}{|c|c|c|c|c|c|c|}
\hline & $\mathrm{ME}$ & RMSE & SNR & GSNR & SS & $\mathrm{CC}$ \\
\hline $\mathrm{ME}$ & 1 & 1 & $1 / 9$ & $1 / 7$ & $1 / 5$ & $1 / 3$ \\
\hline RMSE & 1 & 1 & $1 / 9$ & $1 / 7$ & $1 / 4$ & 1 \\
\hline SNR & 9 & 9 & 1 & 2 & 3 & 7 \\
\hline GSNR & 7 & 7 & $1 / 2$ & 1 & 2 & 5 \\
\hline SS & 5 & 4 & $1 / 3$ & $1 / 2$ & 1 & 2 \\
\hline $\mathrm{CC}$ & 3 & 1 & $1 / 7$ & $1 / 5$ & $1 / 2$ & 1 \\
\hline
\end{tabular}

Table 5: Denoising effect analysis table with different methods.

\begin{tabular}{|c|c|c|c|c|c|c|c|}
\hline Denoising method & Key parameter & ME & RMSE & SNR & GSNR & SS & $\mathrm{CC}$ \\
\hline Db6 wavelet & 1 layer & 0.1116 & 0.1413 & 22.0485 & 1.159 & 0.1955 & 0.9968 \\
\hline Db6 wavelet & 2 layers & 0.0794 & 0.1 & 20.2787 & 1.066 & 0.0284 & 0.9984 \\
\hline Db6 wavelet & 3 layers & 0.0558 & 0.0705 & 19.597 & 1.03 & 0.0038 & 0.9992 \\
\hline Db6 wavelet & 4 layers & 0.0393 & 0.0498 & 19.2912 & 1.014 & 0.0006 & 0.9996 \\
\hline Db6 wavelet & 5 layers & 0.028 & 0.0391 & 19.1149 & 1.005 & 0.0001 & 0.9998 \\
\hline Coif5 wavelet & 1 layer & 0.112 & 0.1413 & 22.0481 & 1.159 & 0.1898 & 0.9968 \\
\hline Coif5 wavelet & 2 layers & 0.0792 & 0.0999 & 20.2753 & 1.066 & 0.027 & 0.9984 \\
\hline Coif5 wavelet & 3 layers & 0.0559 & 0.0704 & 19.5964 & 1.03 & 0.0036 & 0.9992 \\
\hline Coif5 wavelet & 4 layers & 0.0395 & 0.0501 & 19.2905 & 1.014 & 0.0006 & 0.9996 \\
\hline Coif5 wavelet & 5 layers & 0.028 & 0.0378 & 19.1288 & 1.006 & 0.0001 & 0.9998 \\
\hline Sym3 wavelet & 1 layer & 0.1111 & 0.1413 & 22.0504 & 1.159 & 0.2085 & 0.9968 \\
\hline Sym3 wavelet & 2 layers & 0.0783 & 0.0999 & 20.2762 & 1.066 & 0.0332 & 0.9984 \\
\hline Sym3 wavelet & 3 layers & 0.055 & 0.0701 & 19.5914 & 1.03 & 0.0047 & 0.9992 \\
\hline Sym 3 wavelet & 4 layers & 0.0389 & 0.0501 & 19.2925 & 1.014 & 0.0008 & 0.9996 \\
\hline Sym3 wavelet & 5 layers & 0.0276 & 0.0381 & 19.1225 & 1.005 & 0.0002 & 0.9998 \\
\hline Sym4 wavelet & 1 layer & 0.1112 & 0.1412 & 22.0463 & 1.159 & 0.2016 & 0.9968 \\
\hline Sym4 wavelet & 2 layers & 0.0786 & 0.0999 & 20.2735 & 1.066 & 0.0303 & 0.9984 \\
\hline Sym4 wavelet & 3 layers & 0.0553 & 0.0702 & 19.5929 & 1.03 & 0.0041 & 0.9992 \\
\hline Sym4 wavelet & 4 layers & 0.0391 & 0.0499 & 19.2927 & 1.014 & 0.0006 & 0.9996 \\
\hline Sym 4 wavelet & 5 layers & 0.0277 & 0.0373 & 19.1307 & 1.006 & 0.0002 & 0.9998 \\
\hline Sym6 wavelet & 1 layer & 0.1117 & 0.1412 & 22.0465 & 1.159 & 0.1953 & 0.9968 \\
\hline Sym6 wavelet & 2 layers & 0.0794 & 0.1001 & 20.2813 & 1.066 & 0.0285 & 0.9984 \\
\hline Sym6 wavelet & 3 layers & 0.0555 & 0.0701 & 19.5903 & 1.03 & 0.0037 & 0.9992 \\
\hline Sym6 wavelet & 4 layers & 0.039 & 0.0495 & 19.2903 & 1.014 & 0.0006 & 0.9996 \\
\hline Sym6 wavelet & 5 layers & 0.0277 & 0.0372 & 19.1307 & 1.006 & 0.0001 & 0.9998 \\
\hline Low-pass filter & $5 \mathrm{~Hz}$ & 0.0529 & 0.0675 & 18.6924 & 0.983 & 0.0042 & 0.9993 \\
\hline Smoothing filter & 21 & 0.0343 & 0.0436 & 19.2237 & 1.011 & 0.0024 & 0.9997 \\
\hline
\end{tabular}

decision matrix of the TOPSIS method. After the decision matrix is normalized according to Table 5 and equation (15) fd15, the standard weight is obtained from the AHP method. Ideal solution and anti-ideal solution are obtained by equation (16)fd16: the ideal solution is represented by $R+=\{0.0129,0.0028,0.0908,0.0583,4.23 * 10-5,0.0129\}$, and the anti-ideal solution is represented by $R-=\{0.0114,0.0139$, $0.0796,0.0494,0.0698,0.1286\}$. Finally, equation (17)fd17 is used to calculate the distance between the ideal solution and the anti-ideal solution, and the last equation, equation (18) fd18, is used for the final ranking. Based on the $E_{k}$ values shown in Table 6, the application results show that Coif5 wavelet with 3 layers is the best; the $E_{k}$ value is 0.8439 .

\section{Mathematical Model of the FARODE}

This section introduces the analysis steps of FARODE method in objective evaluation for drivability. As shown in Figure 3, it includes the preparation of research objects and indicators, AHP-RS combined weighting method, and multiple hierarchical fuzzy comprehensive evaluation.

4.1. Determination of Research Objects and Objective Indicators. It is important to control the synchronizer, which directly affects the quality of gear shifting performance in the static gearshift process for passenger car with DCT. The optimized shifting force strategy can coordinate with the changes of the synchronizer in real time. After the optimized shifting force is adopted in real vehicles, the shifting time is extended due to the addition of damping force, but it can ensure that the entire shifting time is within the required range. By consulting the expert group, the expected static gearshift quality includes the following attributes: fast, smooth, and comfortable. Therefore, from the three aspects of shifting comfort, response characteristics, and stability, the determination of the drivability evaluation index for static shifting conditions is considered. Figure 4 is the dynamic curve of the subdivided working condition N-D 
TABLE 6: Weight ranking.

\begin{tabular}{|c|c|c|c|c|c|c|c|c|}
\hline Scheme & $E_{k}$ & Rank & Scheme & $E_{k}$ & Rank & Scheme & $E_{k}$ & Rank \\
\hline Db6 (1 layer) & 0.2029 & 25 & Coif5 (5 layers) & 0.8325 & 14 & Sym4 (4 layers) & 0.8384 & 9 \\
\hline Db6 (2 layers) & 0.8006 & 19 & Sym3 (1 layer) & 0.1879 & 27 & Sym4 (5 layers) & 0.8326 & 13 \\
\hline Db6 (3 layers) & 0.8437 & 2 & Sym3 (2 layers) & 0.7858 & 22 & Sym6 (1 layer) & 0.2031 & 24 \\
\hline Db6 (4 layers) & 0.8384 & 6 & Sym3 (3 layers) & 0.8429 & 5 & Sym6 (2 layers) & 0.8002 & 20 \\
\hline Db6 (5 layers) & 0.8318 & 16 & Sym3 (4 layers) & 0.8384 & 8 & Sym6 (3 layers) & 0.8437 & 3 \\
\hline Coif5 (1 layer) & 0.2132 & 23 & Sym3 (5 layers) & 0.8321 & 15 & Sym6 (4 layers) & 0.8384 & 10 \\
\hline Coif5 (2 layers) & 0.805 & 17 & Sym4 (1 layer) & 0.1943 & 26 & Sym6 (5 layers) & 0.8326 & 12 \\
\hline Coif5 (3 layers) & 0.8439 & 1 & Sym4 (2 layers) & 0.795 & 21 & Low-pass $(5 \mathrm{~Hz})$ & 0.8028 & 18 \\
\hline Coif5 (4 layers) & 0.8383 & 7 & Sym4 (3 layers) & 0.8434 & 4 & Smoothing (21) & 0.8348 & 11 \\
\hline
\end{tabular}

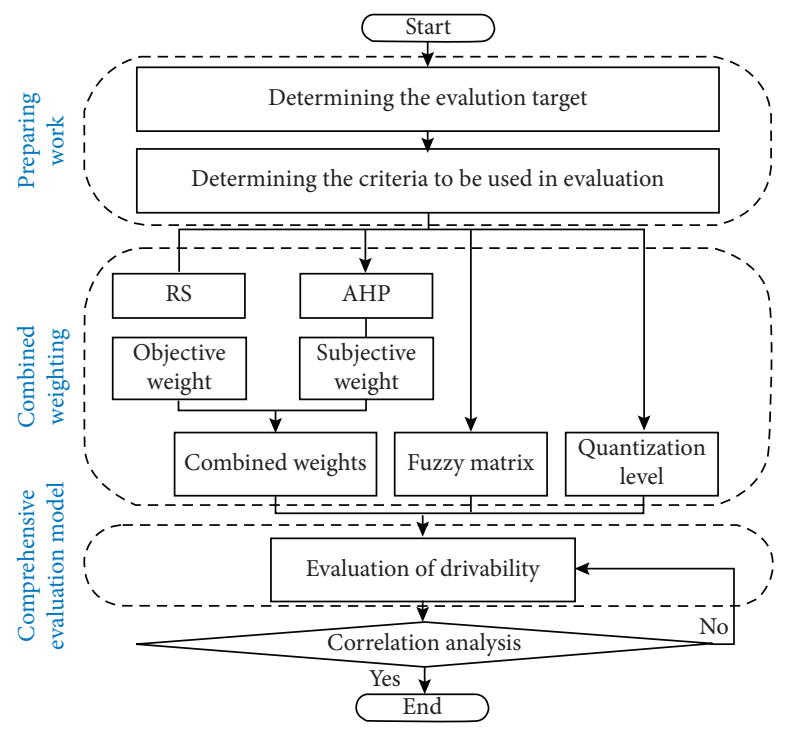

Figure 3: Flowchart of FARODE

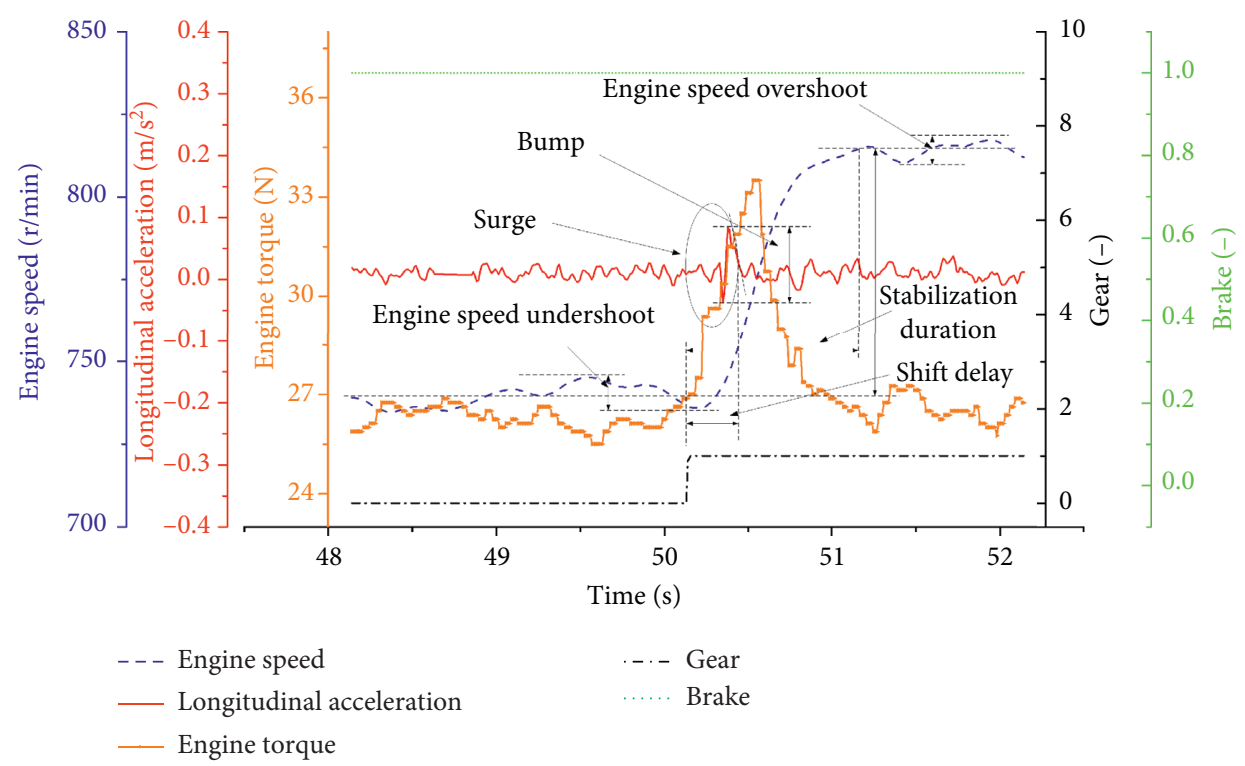

FIGURE 4: Dynamic curves of vehicle performance parameters in static N-D condition.

in the static gearshift processes, and gear position 0 represents $\mathrm{N}$ gear, and gear position 1 represents $D$ gear. In order to achieve the goal of energy saving and smooth clutch integration, OEMs will set different target speeds and target torque outputs when designing EMS and TCU control strategies, which will affect the driving experience of drivers 
and passengers. In the $N$ gear, the clutch is in a disconnected state, no transmission function is needed, and a lower target engine speed and engine torque is usually set. However, the clutch is engaged in $D$ gear, and the engine speed and engine torque are compensated by EMS or TCU control strategy, which is used to ensure that the surge for vehicle is slight.

As shown in Figure 4, the response characteristics of the vehicle consider the effects of shift delay (SD) and shift stabilization duration (SSD); the stability characteristics of the vehicle consider engine speed overshoot (ESO), and engine speed undershoot (ESU). Longitudinal acceleration bump and surge of static gearshift are used to describe the shift comfort of the vehicle.

According to the SAE (J1441) scoring standard and the characteristics of static gear shift conditions, the quantization level score is shown in Table 7. The quantization level of drivability in static gearshift conditions is set to five levels. The indicator classification criteria are shown in Table 8.

4.2. AHP-RS Combined Weighting Method. The index weight and rationality in the objective evaluation of vehicle drivability have an important influence on the reliability of the prediction results, which is affected by the cognition of the evaluator and the dynamic characteristics for vehicles. The perception for evaluator is strongly subjective and usually used for subjective empowerment. The vehicle dynamic characteristics are reflected in real and objective driving data, which are usually used for objective weight calculation. In order to be able to take into account both the cognition for evaluator and the vehicle dynamic characteristics, it is necessary to establish a subjective and objective weight calculation model. The principle of minimum information entropy is used to determine the subjective and objective allocation weights, which are applied to objective evaluation model of drivability for vehicles.

Subjective weights are based on prior knowledge and experience. The subjective weight $s(i)$ of the lowest-level indicator relative to the highest-level indicator is determined based on AHP model in the principle of Section 3.2.

Objective weights are determined by RS principle based on the information from original data and the logic of knowledge system. The traditional RS method can only express the information of current data on decision and ignore the prior knowledge of decision-makers. Based on Table 8, an improved RS method is developed to calculate the weight of drivability evaluation index. The objective weight $o(i)$ of each evaluation index is calculated by

$$
\begin{aligned}
\Phi_{A}(B) & =\frac{1}{m} \sum_{i=1}^{n}\left|\Phi_{A}\left(B_{i}\right)\right|, \\
\Phi_{A-A_{i}}(B) & =\frac{1}{m} \sum_{i=1}^{n}\left|\Phi_{A-A_{i}}\left(B_{i}\right)\right|, \\
o(i) & =\frac{\Phi_{A}(B)-\Phi_{A-A_{i}}(B)}{\sum_{i=1}^{n}\left|\Phi_{A}(B)-\Phi_{A-A_{i}}(B)\right|},
\end{aligned}
$$

TABLE 7: Drivability quantification level.

\begin{tabular}{lcc}
\hline Subjective description & Rating interval & Grade rating \\
\hline Well & $(8,10]$ & 9 \\
Better & $(7,8]$ & 7.5 \\
General & $(5,7]$ & 6 \\
Poor & $(4,5]$ & 4.5 \\
Difference & $(2,4]$ & 3 \\
\hline
\end{tabular}

where $m$ is the number of samples, $\Phi_{A}(B)$ and $\Phi_{A}\left(B_{i}\right)$ are the dependence and the number of compatible samples in the decision table, respectively, and $\Phi_{A-A_{i}}(B)$ and $\Phi_{A-A_{i}}\left(B_{i}\right)$ are the dependence and the number of compatible samples after excluding the evaluation index $A_{i}$, respectively.

In order to seek the balance between subjective and objective weights, the principle of minimum relative information entropy is used to reduce the deviation between subjective and objective weights, and a more scientific and reliable AHP-RS combination optimization weight $w$ is obtained by

$$
w(i)=\frac{\sqrt{s(i) o(i)}}{\sum_{i=1}^{n} \sqrt{s(i) o(i)}} .
$$

4.3. Multilevel Fuzzy Comprehensive Evaluation Model. In order to solve the uncertainty of subjective evaluation results, fuzzy mathematics theory is applied to build the comprehensive evaluation model. According to the review standard (Table 7) and the quantitative grade of the static gearshift drivability evaluation index (Table 8), the trapezoid distribution membership function is selected to determine the membership grade. The distribution membership function is shown in Figure 5; the shaded area in the figure is the fuzzy interval. The limits of fuzzy interval $c_{i}, i=1,2, \ldots$, 10 , are obtained by analysing the real vehicle test results. The membership function is expressed by

$$
\begin{aligned}
& \mu_{1}=\left\{\begin{array}{l}
1, \quad c_{1} \leq x \leq c_{2}, \\
\frac{c_{3}-x}{c_{3}-c_{2}}, \quad c_{2}<x<c_{3}, \\
0, \quad x \geq c_{3},
\end{array}\right. \\
& \mu_{k}=\left\{\begin{array}{l}
\frac{x-c_{2(k-1)}}{c_{2 k-1}-c_{2(k-1)}}, \quad c_{2(k-1)}<x<c_{2 k-1}, \\
1, \quad c_{2 k-1} \leq x \leq c_{2 k}, \\
\frac{c_{2 k+1}-x}{c_{2 k+1}-c_{2 k}}, \quad c_{2 k}<x<c_{2 k+1}, \\
0, \quad x<c_{2(k-1)}, x \geq c_{2 k+1},
\end{array}\right. \\
& \mu_{5}=\left\{\begin{array}{l}
0, \quad x<c_{8}, \\
\frac{x-c_{8}}{c_{9}-c_{8}}, \quad c_{8} \leq x \leq c_{9}, \\
1, \quad x>c_{9},
\end{array}\right.
\end{aligned}
$$


TABLE 8: Indicator classification criteria.

\begin{tabular}{lcccccc}
\hline Level & SD & SSD & ESO & ESU & Bump & Surge \\
\hline Well & {$[0,0.3)$} & {$[0,0.5)$} & {$[0,20)$} & {$[0,20)$} & {$[0,0.05)$} & {$[0,0.03)$} \\
Better & {$[0.3,0.5)$} & {$[0.5,1.0)$} & {$[20,30)$} & {$[20,30)$} & {$[0.05,0.075)$} & {$[0.03,0.045)$} \\
General & {$[0.5,0.8)$} & {$[1.0,1.5)$} & {$[30,50)$} & {$[30,50)$} & {$[0.075,0.125)$} & {$[0.045,0.075)$} \\
Poor & {$[0.8,1.0)$} & {$[1.5,2.0)$} & {$[50,60)$} & {$[50,60)$} & {$[0.125,0.15)$} & {$[0.075,0.09)$} \\
Difference & {$[1,+\infty)$} & {$[2,+\infty)$} & {$[60,+\infty)$} & {$[60,+\infty)$} & {$[0.15,+\infty)$} & {$[0.09,+\infty)$} \\
\hline
\end{tabular}

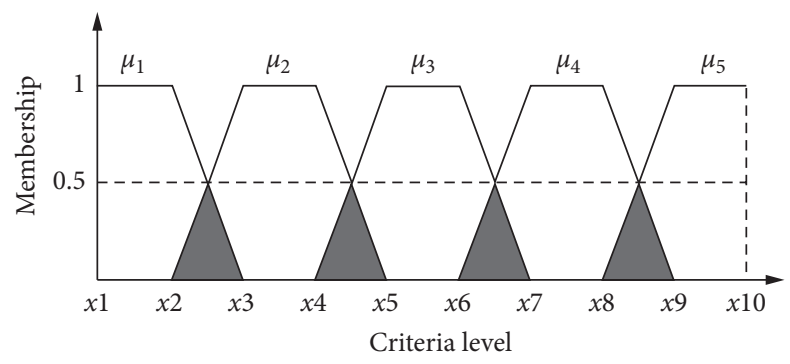

Figure 5: The membership function of the evaluation indexes relative to all levels.

where $k=2,3,4 ; \mu_{\mathrm{i}} \in[0,1](i=1,2,3,4,5)$. It is the probability of different membership function.

The fuzzy matrix $M=\left(\mu_{k j}\right)_{5 \times n}$ is obtained, and the score is quantization level $S=\left[\begin{array}{lllll}9 & 7.5 & 6 & 4.5 & 3\end{array}\right]$. According to $M$, $S$, and the optimization weight $w$, the objective score can be calculated as $T=S \cdot M \cdot w^{T}$.

\section{Case Study of Objective Drivability Evaluation}

5.1. Case Study. In this section, I-DOET is used to collect and process data on 15 static gearshift conditions of passenger cars with DCT on the Chinese market, as shown in Figure 6, including I-DOET, an acceleration sensor, and a laptop. In order to test the signals of the vehicle's dynamic response accurately, the acceleration sensor is installed on the driver's seat rail through the $\mathrm{F}$ clip, and its position is close to the centre of mass of the vehicle. Multisource signal is obtained by using different signal sources in parallel acquisition control method, and the signal sampling rate, signal type, data acquisition, and data storage can be realized by the designed and written digital acquisition system. The static gearshift condition of real vehicle test is designed as follows: windows, air condition, and other electrical equipment are turned off, engine is started and the engine speed is idling, hand brake is released while brake system is depressed, and gear is shifted at norm speed, including P-D, D-N, N-R, R-N, N-D, D-R, R-D, D-P, P-R, and R-P. Meanwhile, gearbox gears, engine speed, engine torque, vehicle longitudinal acceleration, and braking signals are obtained by I-DOET hardware and laptop.

In this real vehicle test, the sampling frequency of each signal is set as $100 \mathrm{~Hz}$, and the static gearshift conditions of 15 vehicles are collected and given the subjective scores by 3 experts. According to the analysis results ofSection 3, the 3layer Coif5 wavelet threshold method is used for denoising of the longitudinal acceleration signal. Sliding window method and D-S semantic segmentation method are used for working condition recognition and objective index extraction, respectively. 43 of the 45 sets of effective objective indexes are used for model training and weighting. Table 9 lists the remaining two sets of indicator data $\left(N_{1}\right.$ and $\left.N_{2}\right)$ used to verify the accuracy of proposed FCA model.

5.2. Discussion. In order to obtain the subjective weights more accurately, in this study, an expert group composed of two scholars from Wuhan University of Technology and three vehicle calibration engineers from Dongfeng Motor Corporations Technical Centre is formed. A hierarchical model for objective evaluation of drivability in static gearshift condition is constructed on the basis of prior knowledge of the expert group, as shown in Figure 7.

The expert group determined the indicators to be used in the static gearshift evaluation model and compared the criteria in pairs. Table 2 is used to compare the relative advantages and disadvantages of the evaluation indicators by the expert group, and the judgment matrix of the target layer and the subtarget layer is established in Tables 10 and 11.

The subjective weights of the target layer and subtarget layer in static shifting conditions are obtained by the AHP method, and the objective weights of the subtarget layer are calculated by the RS method. The optimization weight is calculated by equation (20)fd20.The subjective weight of the target layer and the optimized weight distribution of the subtarget layer are shown in Table 12.

Figure 8 compares the predicted results of the drivability evaluation based on FARODE model and FAHP method, including the objective scores of FARODE and FAHP with IDs $\mathrm{N}_{1}$ and $\mathrm{N}_{2}$ and the real scores given by the expert group. From the subjective and objective score analysis in Figures $8(\mathrm{a})$ and $8(\mathrm{~b})$, the prediction results of FARODE and FAHP for static gearshift subworking conditions are demonstrated. The scoring results show that the drivability score 


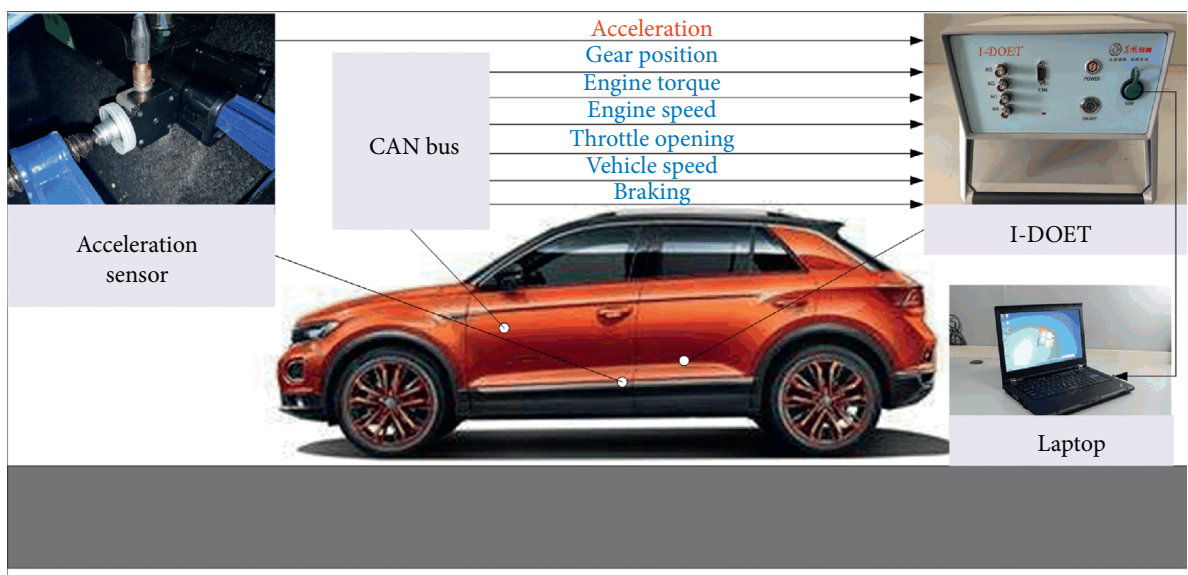

Random road

Figure 6: Real vehicle test.

TABLE 9: Evaluation indexes values for ID $N_{1}$ and ID $N_{2}$.

\begin{tabular}{|c|c|c|c|c|c|c|c|}
\hline Indicator data & Operation model & $\mathrm{SD}$ & SSD & ESO & ESU & Bump & Surge \\
\hline \multirow{10}{*}{$N_{1}$} & P-D & 0.32 & 0.53 & 27.158 & 29.132 & 0.062 & 0.043 \\
\hline & $\mathrm{D}-\mathrm{N}$ & 0.48 & 0.87 & 30.51 & 25.802 & 0.065 & 0.042 \\
\hline & $\mathrm{N}-\mathrm{R}$ & 0.37 & 0.69 & 23.289 & 23.097 & 0.073 & 0.047 \\
\hline & $\mathrm{R}-\mathrm{N}$ & 0.39 & 0.53 & 28.283 & 23.612 & 0.059 & 0.037 \\
\hline & N-D & 0.32 & 0.53 & 27.796 & 23.447 & 0.062 & 0.043 \\
\hline & $\mathrm{D}-\mathrm{R}$ & 0.56 & 0.89 & 24.798 & 27.178 & 0.086 & 0.06 \\
\hline & R-D & 0.43 & 0.81 & 23.796 & 22.289 & 0.072 & 0.046 \\
\hline & D-P & 0.22 & 0.51 & 19.191 & 23.75 & 0.062 & 0.048 \\
\hline & P-R & 0.35 & 0.62 & 21.197 & 16.763 & 0.075 & 0.043 \\
\hline & $\mathrm{R}-\mathrm{P}$ & 0.36 & 0.61 & 24.987 & 18.02 & 0.071 & 0.05 \\
\hline \multirow{10}{*}{$N_{2}$} & P-D & 0.35 & 0.89 & 28.671 & 20.467 & 0.076 & 0.048 \\
\hline & $\mathrm{D}-\mathrm{N}$ & 0.28 & 0.51 & 27.767 & 24.559 & 0.054 & 0.045 \\
\hline & $\mathrm{N}-\mathrm{R}$ & 0.54 & 0.92 & 32.3 & 21.453 & 0.072 & 0.05 \\
\hline & $\mathrm{R}-\mathrm{N}$ & 0.47 & 0.66 & 24.453 & 18.842 & 0.075 & 0.038 \\
\hline & N-D & 0.34 & 0.55 & 27.849 & 22.954 & 0.058 & 0.041 \\
\hline & $\mathrm{D}-\mathrm{R}$ & 0.62 & 0.99 & 10.079 & 18.194 & 0.096 & 0.062 \\
\hline & R-D & 0.51 & 0.85 & 17.241 & 16.91 & 0.078 & 0.05 \\
\hline & D-P & 0.27 & 0.64 & 24.25 & 24.237 & 0.062 & 0.048 \\
\hline & P-R & 0.38 & 0.71 & 13.941 & 18.493 & 0.084 & 0.047 \\
\hline & R-P & 0.43 & 0.76 & 29.586 & 28.875 & 0.064 & 0.044 \\
\hline
\end{tabular}

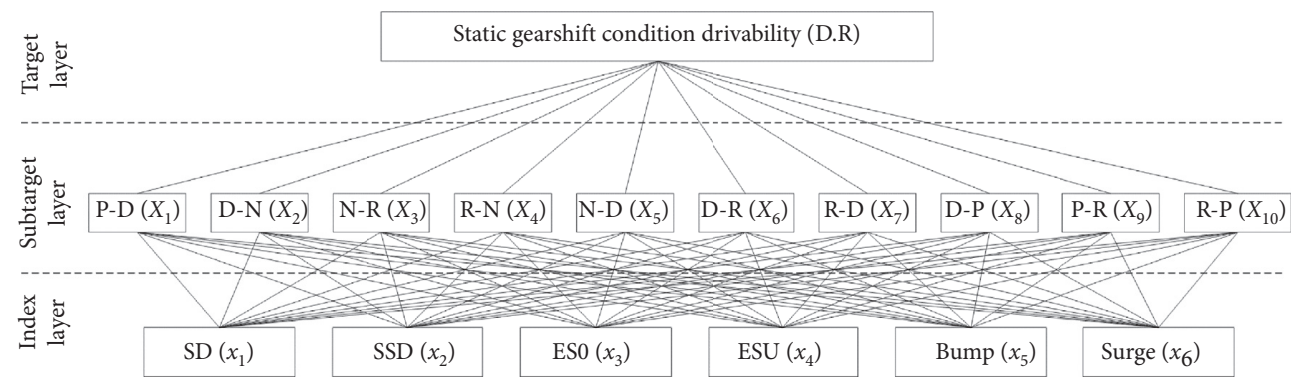

FIgURE 7: The hierarchical model of drivability in static gearshift condition.

of D-R subdivision operating conditions in static gearshift is lower than other suboperating conditions, which is closer to the expert group score and consistent with the actual vehicle response. This is related to the driver needs to control the filling and discharging of oil during the clutch disengagement and coupling action during the D-R shift operation. The formulation of the TCU control strategy needs to consider the shift completion time and shift shock 
TABLE 10: The judgment matrix for the target layer.

\begin{tabular}{ccccccccccc}
\hline & $X_{1}$ & $X_{2}$ & $X_{3}$ & $X_{4}$ & $X_{5}$ & $X_{6}$ & $X_{7}$ & $X_{8}$ & $X_{9}$ & $X_{10}$ \\
\hline$X_{1}$ & 1 & 2 & 2 & 2 & 2 & $1 / 2$ & $1 / 2$ & 1 & 1 \\
$X_{2}$ & $1 / 2$ & 1 & 1 & 1 & 1 & $1 / 3$ & $1 / 3$ & $1 / 2$ & $1 / 2$ \\
$X_{3}$ & $1 / 2$ & 1 & 1 & 1 & 1 & $1 / 3$ & $1 / 3$ & $1 / 2$ & $1 / 2$ \\
$X_{4}$ & $1 / 2$ & 1 & 1 & 1 & 1 & $1 / 4$ & $1 / 4$ & $1 / 2$ & $1 / 2$ \\
$X_{5}$ & $1 / 2$ & 1 & 1 & 1 & 1 & $1 / 4$ & $1 / 4$ & $1 / 2$ & $1 / 2$ \\
$X_{6}$ & 2 & 3 & 3 & 4 & 4 & 1 & 1 & 2 & $1 / 2$ \\
$X_{7}$ & 2 & 3 & 3 & 4 & 4 & 1 & 1 & 2 & 2 \\
$X_{8}$ & 1 & 2 & 2 & 2 & 2 & $1 / 2$ & $1 / 2$ & 1 & 1 \\
$X_{9}$ & 1 & 2 & 2 & 2 & 2 & $1 / 2$ & $1 / 2$ & 1 & 1 \\
$X_{10}$ & 1 & 2 & 2 & 2 & 2 & $1 / 2$ & $1 / 2$ & 1 & 1 \\
\hline
\end{tabular}

TABLE 11: The judgment matrix for the subtarget layer.

\begin{tabular}{ccccccc}
\hline & $x_{1}$ & $x_{2}$ & $x_{3}$ & $x_{4}$ & $x_{5}$ & $1 / 3$ \\
\hline$x_{1}$ & 1 & 1 & 2 & 2 & 2 & $1 / 3$ \\
$x_{2}$ & 1 & 1 & 2 & 1 & $1 / 7$ \\
$x_{3}$ & $1 / 2$ & $1 / 2$ & 1 & 1 & $1 / 3$ & $1 / 3$ \\
$x_{4}$ & $1 / 2$ & $1 / 2$ & 1 & 7 & 1 & $1 / 7$ \\
$x_{5}$ & 3 & 3 & 7 & 7 & 1 \\
$x_{6}$ & 3 & 3 & 7 & & 1 \\
\hline
\end{tabular}

TABLE 12: Weight distribution of the target layer and subtarget layer.

\begin{tabular}{|c|c|c|c|c|c|c|c|}
\hline \multirow{2}{*}{ Operation model } & \multicolumn{6}{|c|}{ Subtarget weight } & \multirow{2}{*}{$\begin{array}{c}\text { Target weight } \\
w\end{array}$} \\
\hline & $w_{1}$ & $w_{2}$ & $w_{3}$ & $w_{4}$ & $w_{5}$ & $w_{6}$ & \\
\hline$X_{1}$ & 0.1447 & 0.1390 & 0.1033 & 0.0998 & 0.2470 & 0.2662 & 0.1015 \\
\hline$X_{2}$ & 0.1385 & 0.1281 & 0.1022 & 0.0883 & 0.2634 & 0.2795 & 0.054 \\
\hline$X_{3}$ & 0.1383 & 0.1320 & 0.0954 & 0.0995 & 0.2566 & 0.2782 & 0.054 \\
\hline$X_{4}$ & 0.1403 & 0.1330 & 0.0812 & 0.0865 & 0.2733 & 0.2857 & 0.0508 \\
\hline$X_{5}$ & 0.1362 & 0.1274 & 0.0939 & 0.0879 & 0.2707 & 0.2839 & 0.0508 \\
\hline$X_{6}$ & 0.138 & 0.1293 & 0.0891 & 0.0823 & 0.2606 & 0.3007 & 0.1923 \\
\hline$X_{7}$ & 0.1457 & 0.1372 & 0.0949 & 0.0886 & 0.2589 & 0.2747 & 0.1923 \\
\hline$X_{8}$ & 0.1449 & 0.1326 & 0.0957 & 0.0999 & 0.2686 & 0.2583 & 0.1015 \\
\hline$X_{9}$ & 0.1306 & 0.1221 & 0.1008 & 0.0901 & 0.2718 & 0.2846 & 0.1015 \\
\hline$X_{10}$ & 0.1399 & 0.1328 & 0.1012 & 0.0916 & 0.2608 & 0.2737 & 0.1015 \\
\hline
\end{tabular}

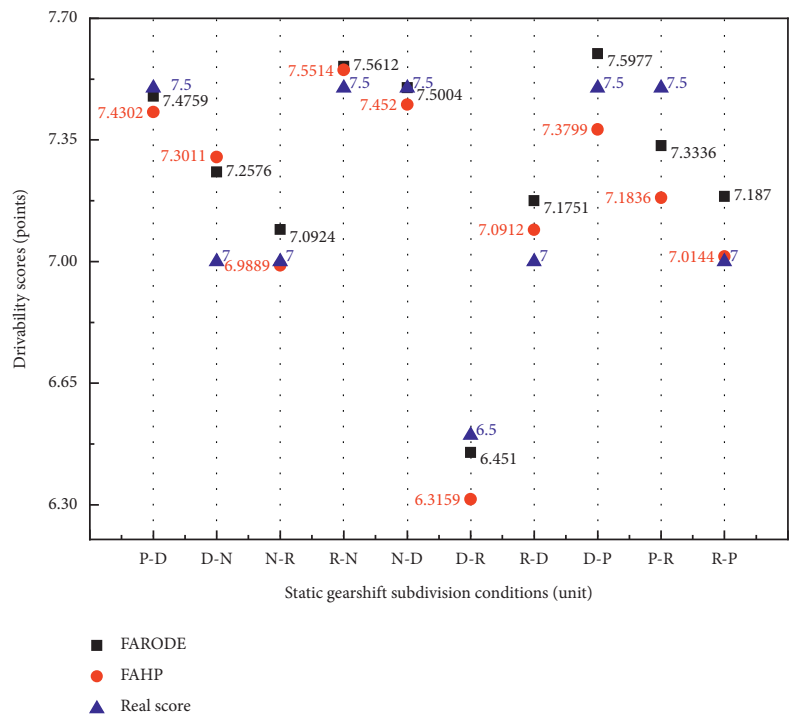

(a)

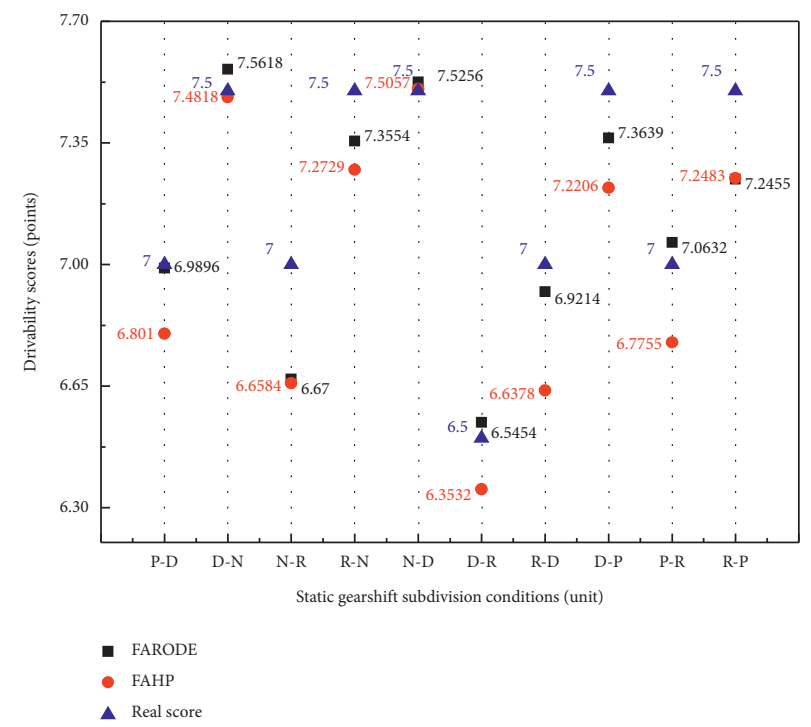

(b)

FIgURE 8: Scoring results and real score of different drivability evaluation model. (a) ID $N_{1 ;}$ (b) ID $N_{2}$. 
TABLE 13: Comparison indexes for different evaluation models.

\begin{tabular}{lcccc}
\hline & \multicolumn{2}{c}{ ID $N_{1}$} & \multicolumn{2}{c}{ ID $N_{2}$} \\
& FARODE & FAHP & FARODE & FAHP \\
\hline Maximum deviation & 0.2576 & 0.3164 & 0.33 & 0.36 \\
Correlation coefficient & 0.9323 & 0.8893 & 0.921 & 0.8548 \\
$\begin{array}{l}\text { Errors more than 0.25/ } \\
\text { time }\end{array}$ & 1 & 2 & 2 & 4 \\
Pass rate & $90 \%$ & $80 \%$ & $80 \%$ & $60 \%$ \\
\hline
\end{tabular}

simultaneously. The calibration of D-R subdivision conditions has high requirements. Thus, the effectiveness of the FARODE model and the FAHP method in the evaluation of the drivability under static gearshift conditions is verified.

The accuracy and stability of the drivability evaluation model are analysed by introducing comparative indicators including maximum deviation, Pearson correlation coefficient, and pass rate. Looking at Table 13, the maximum deviation of the FARODE comprehensive evaluation model in the ID $N_{1}$ and ID $N_{2}$ static gearshift conditions in actual vehicle testing is 0.2576 and 0.33 , respectively. The maximum deviation of the AHP method is 0.3164 and 0.36, respectively. The correlation between the model prediction results and the real score of the expert group is researched through Pearson correlation coefficients to determine the model accuracy. The Pearson correlation coefficients of the FAHP method are 0.83 and 0.8548 , respectively, and the FARODE model can increase the Pearson correlation coefficients to 0.9323 and 0.921 . With reference to the scoring rules of the subjective evaluation engineers of Dongfeng Motor Centre and the sensitivity test results in [23], it is assumed that when the predicted result and the actual score exceed 0.25 , the predicted result of the model needs to be improved. The adoption rate of FARODE mathematical model for ID $N_{1}$ and ID $N_{2}$ is $90 \%$ and $80 \%$, respectively. However, the passing rate through FAHP can only reach $80 \%$ and $60 \%$. The analysis results show that drivability evaluation of the FARODE model in static gearshift conditions is more accurate and reliable than the simple FAHP method. FARODE model is used to obtain the drivability evaluation scores in static gearshift with ID $\mathrm{N}_{1}$ and ID $\mathrm{N}_{2}$, and the scores are 7.1641 and 7.0234, respectively. Analysing the subdivision conditions that affect the static gearshift and their objective indicators can be used to guide the improvement of drivability for automobiles.

\section{Conclusions}

This paper developed an intelligent drivability objective evaluation tool (I-DOET) for passenger cars with dualclutch transmission (DCT) and verified by real vehicle testing. Based on the analytic hierarchy process (AHP) and technique for order preference by similarity to ideal solution (TOPSIS), the signal denoising method and its key parameters suitable for drivability evaluation are selected. Thereafter, combined with the uncertainty characteristics of subjective judgments, a mathematical model of the objective drivability evaluation FARODE (fuzzy AHP-RS based on objective drivability evaluation) is proposed by using the fuzzy comprehensive assessment (FCA) method. Furthermore, the static gearshift condition is taken as a case study to verify the accuracy and stability of evaluation model including FARODE and FAHP. The conclusions of this investigation are given as follows:

(1) Based on AHP-TOPSIS model, the ME, RMSE, SNR, SNRG, SS, and CC are selected as index layers to take the best scheme of denoising. The application results show that Coif5 wavelet with 3 layers is the most suitable de-noising method for objective evaluation of drivability.

(2) FARODE method is introduced in objective evaluation for drivability; it includes the preparation of research objects and indicators, AHP-RS combined weighting method, and multiple hierarchical fuzzy comprehensive evaluation. The AHP and rough set (RS) method are used to calculate the subjective and objective weights of the drivability evaluation, respectively. The principle of minimum relative information entropy is used to solve the unscientific problem of subjective and objective weight distribution.

(3) The static gearshift condition focused on by the subjective evaluation experts is taken as a case study, and maximum deviation, Pearson correlation coefficient, and pass rate are used as comparative indicators to compare the FARODE evaluation model and the FAHP method. The real vehicle test results show that the FARODE model proposed in this paper is more accurate and reliable.

Given the recommendations for the follow-up investigations, the fuzzy membership function of objective indicators should be further studied with respect to the stability and accuracy of the consistency of subjective and objective evaluations. Moreover, future investigations are needed to find a more suitable drivability evaluation method in mathematics.

\section{Data Availability}

The data used to support the findings of this study are available from the corresponding author upon request.

\section{Conflicts of Interest}

The authors declare that they have no conflicts of interest.

\section{Acknowledgments}

The authors would like to express their deep appreciation to Hubei Key Laboratory of Advanced Technology for Automotive Components for the continuous support. The authors also acknowledge the support of Hubei Collaborative Innovation Centre for Automotive Components Technology and Dongfeng Motor Corporations Technical Centre. This work was financially supported in part by the National Natural Science Foundation of the People's Republic of China (grant number: 51505354). 


\section{References}

[1] W. S. Chang, C. Jongdae, W. C. Suk, and L. Wonsik, "An objective method of drivability evaluation using a simulation model for hybrid electric vehicles," International Journal of Precision Engineering and Manufacturing, vol. 15, no. 2, pp. 219-226, 2014.

[2] K. Chandrasekaran, N. Rao, S. Palraj, and C. Kurella, "Objective drivability evaluation on compact suv and comparison with subjective drivability," SAE, Pittsburgh, PA, USA, SAE Technical Paper 2017-26-0153, 2017.

[3] Z. F. Chen and X. M. Shi, The Subjective Evaluation of Vehicle Dynamic Performance, China: People's Communication Press, Beijing, China, 2011.

[4] P. Schoeggl and E. Ramschak, Vehicle Drivability Assessment Using Neural Networks For Development, Calibration And Quality Tests, SAE Technical Paper, Pittsburgh, PA, USA, 2000.

[5] AVL-DRIVE Function Description, Advanced Software Version 3x, 2007.

[6] H. Jayaraman, N. Rao, S. Muthiah et al., "Optimization of tipin response character of sports utility vehicle and verification with objective methodology," Neuropeptides, vol. 18, no. 2, pp. 87-91, 2015.

[7] M. Khodabakhshian, L. Feng, and J. Wikander, Optimization of Gear Shifting and Torque Split for Improved Fuel Efficiency and Drivability of HEVs, SAE World Congress and Exhibition, Detroit, MI, USA, 2013.

[8] W. Zhou, X. X. Guo, X. F. Pei, and C. C. Zhang, "Research on objective drivability evaluation with multi-source information fusion for passenger car," SAE, Pittsburgh, PA, USA, SAE Technical Paper 2020-01-1044, 2020.

[9] T. Deng, C. Lin, J. Luo, and B. Chen, "NSGA-II multi-objectives optimization algorithm for energy management control of hybrid electric vehicle," Proceedings of the Institution of Mechanical Engineers, Part D: Journal of Automobile Engineering, vol. 233, no. 4, pp. 1023-1034, 2019.

[10] W. S. Chang, K. Hyungkyoon, K. K. Mun, L. Wonsik, and W. C. Suk, "Development of an evaluation method for quantitative drivability in heavy-duty vehicles," Journal of Mechanical Science and Technology, vol. 28, no. 5, pp. 16151621, 2014.

[11] E. Galvagno, D. Morina, A. Sorniotti, and M. Velardocchia, "Drivability analysis of through-the-road-parallel hybrid vehicles," Meccanica, vol. 48, no. 2, pp. 351-366, 2013.

[12] S. Lakshmanan, A. Palaniappan, and V. Chekuri, "Methodology for evaluation of drivability attributes in commercial vehicle," SAE, Pittsburgh, PA, USA, SAE Technical Paper 2015-01-2767, 2015.

[13] X. Zhao, "Research on gearshift control for dual clutch transmission based on objective evaluation," $\mathrm{Ph} \mathrm{D}$. thesis, Jilin University, Changchun, China, 2015.

[14] S. G. Pickering and C. J. Brace, "Automated data processing and metric generation for driveability analysis," Proceedings of the Institution of Mechanical Engineers, Part D: Journal of Automobile Engineering, vol. 221, no. 4, pp. 429-441, 2007.

[15] C. H. Jiang, S. Chen, Y. W. Chen, and B. Y. Zhang, "A MEMS IMU de-noising method using long short term memory recurrent neural networks (LSTM-RNN)," Sensors, vol. 18, no. 10, pp. 1-14, 2018.

[16] A. S. EI-Wakeel, A. Osman, and N. Zorba, "Robust positioning for road information services in challenging environments," IEEE Sensors Journal, vol. 20, no. 6, pp. 3182-3195, 2020.
[17] W. Seo, S. Hwang, J. Park, and J.-M. Lee, "Precise outdoor localization with a GPS-INS integration system," Robotica, vol. 31, no. 3, pp. 371-379, 2013.

[18] C. Zhang and C. Y. Xu, "A de-noising method of acceleration signal for vehicle based on kalman filter and average filter," Advanced Materials Research, vol. 225-226, no. 1-2, pp. 605-608, 2011.

[19] Q. K. Zhao, C. G. Liu, X. R. Gao, and L. Luo, "Improved wavelet de-noising method of rail vibration signal for wheel tread detection," Seventh International Symposium on Precision Engineering Measurements and Instrumentation, vol. 8321, pp. 1-7, 2011.

[20] H. J. Liu, M. Li, W.. Huang, and R. H. Tong, "Signal de-noising method for whole vehicle drivability evaluation based on wavelet transform," Noise and Vibration Control, vol. 38, no. 1, pp. 103-108, 2018.

[21] H. J. Liu, S. G. Liu, and M. Li, "EMD and wavelet threshold denoising method of gear-shift acceleration signals," Noise and Vibration Control, vol. 38, no. 2, pp. 198-203, 2018.

[22] F. Xiao, G. S. Chen, W. Zatar, and J. L. Hulsey, "Signature extraction from the dynamic responses of a bridge subjected to a moving vehicle using complete ensemble empirical mode decomposition," Journal of Low Frequency Noise, Vibration and Active Control, pp. 1-17, 2019.

[23] C. Jauch, S. Tamilarasan, and K. Bovee, "Modeling for drivability and drivability improving control of HEV," Control Engineering Practice, vol. 70, pp. 50-62, 2018.

[24] C. Jauch, S. Tamilarasan, and K. Bovee, "Design and verification of drivability improving control fro the Eco-CAR 2 hybrid electric vehicle," in Proceedings of the 2016 American Control Conference, pp. 631-636, Boston, MA, USA, July 2016.

[25] K. Koprubasi, E. R. Westervelt, and G. Rizzoni, "Experimental validation of a model for the control of drivability in a hybridelectric vehicle," in Proceedings of the ASME International Mechanical Engineering Congress and Exposition, vol. 16, pp. 105-114, Seattle, WA, USA, November 2007.

[26] W. Huang, H. J. Liu, and Y. F. Ma, "Drivability evaluation model using principal component analysis and optimized extreme learning machine," Journal of Vibration and Control, vol. 25, no. 6, pp. 2274-2281, 2019.

[27] L. Y. Liang, S. Chen, and P. Li, The Evaluation of Vehicle Interior Impact Noise Inducing by Speed Bumps Based on Multi-Features Combination and Support Vector Machine, , pp. 1-21, Applied Acoustics, 2020.

[28] Y. Fu, Y. Lei, S. H. Shao, and H. Zeng, "Shift quality evaluation of DCT based on TOPSIS model," SAE, Pittsburgh, PA, USA, SAE Technical Paper 2014-01-1166, 2014.

[29] P. Schoeggl and E. Ramschak, "Vehicle drivability assessment using neural networks for development, calibration and quality tests," SAE, Pittsburgh, PA, USA, SAE Technical Paper 2000-01-0702, 2000.

[30] W. Huang and H. J. Liu, "Application of fuzzy dynamic weights drivability evaluation model in tip-in condition," Journal of Vibration and Control, vol. 25, no. 4, pp. 1-9, 2018.

[31] K. S. Leung, H. B. Ji, and L. Yee, "Adaptive weighted outerproduct learning associative memory," IEEE Transactions on System, Man, and Cybernetics, Part B, Cybernetics, vol. 27, no. 3, pp. 533-543, 1997.

[32] L. Zhou, Q. X. Yu, and D. Z. Liu, "Compressive sensing-based vibration signal reconstruction using sparsity adaptive subspace pursuit," Advances in Mechanical Engineering, vol. 10, no. 8, pp. 1-12, 2018. 